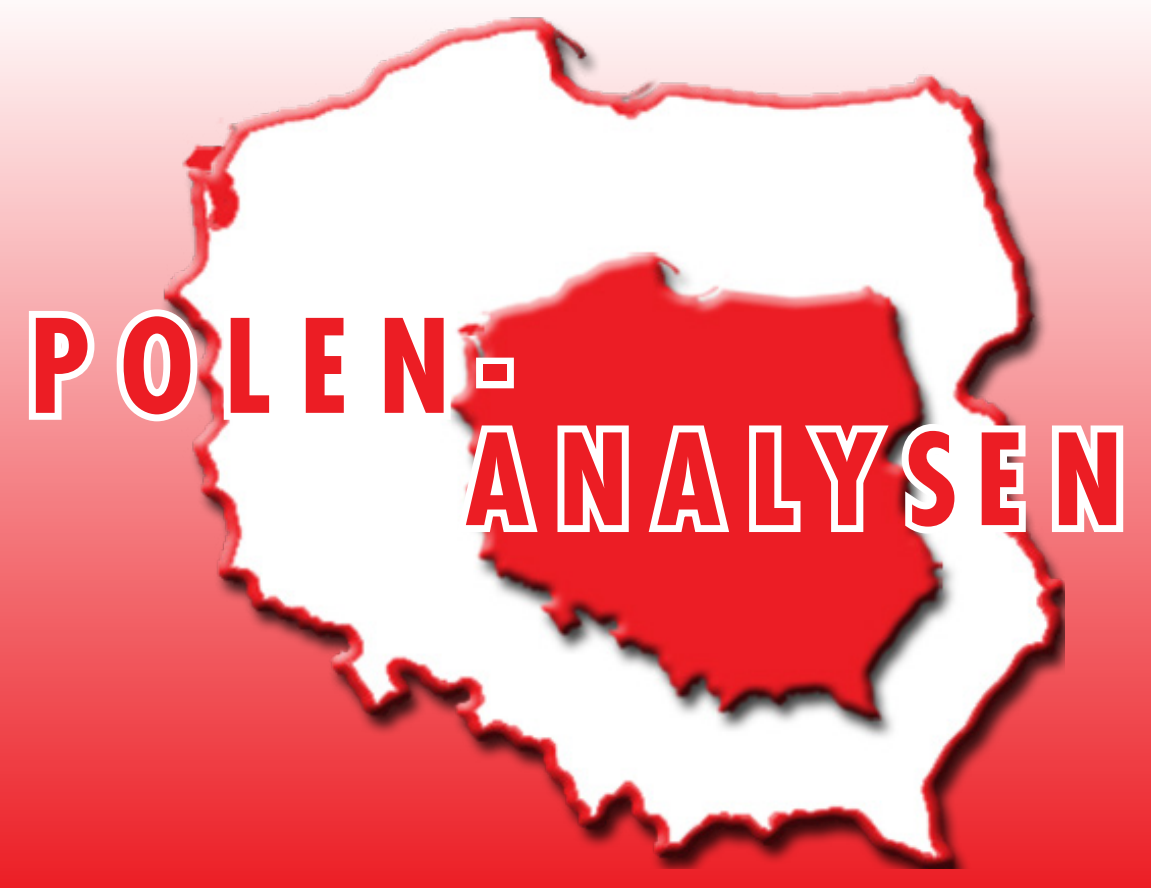

www.laender-analysen.de/polen

\title{
EINE PROTESTBEWEGUNG IN POLEN
}

ANALYSE

Die Antiregierungsproteste in Polen

Mateusz Fałkowski, Warschau

\section{- UMFRAGE}

Bewertung der politischen Situation

CHRONIK

7. - 20. Juni 2016

Herausgegeben mit finanzieller Unterstützung

der Stiftung für deutsch-polnische Zusammenarbeit

FUNDACJA WSPÓŁPRACY

POLSKO-NIEMIECKIEJ

STIFTUNG

FÜR DEUTSCH-POLNISCHE

ZUSAMMENARBEIT

\section{DEUTSCHES POLEN INSTITUT}

- Forschungsstelle Osteuropa an der Universität Bremen
Deutsche Gesellschaft

DGO für Osteuropakunde e.V. 


\section{Die Antiregierungsproteste in Polen}

Mateusz Fałkowski, Warschau

\section{Zusammenfassung}

Seit Jahren wird die polnische parlamentarische Politik immer wieder von Protestpolitik auf der Straße begleitet. In der Regierungszeit der Bürgerplattform (Platforma Obywatelska - PO), insbesondere seit dem Jahr 2010, organisierten rechte, mit Recht und Gerechtigkeit (Prawo i Sprawiedliwość - PiS) und den Nationalisten verbundene Kreise regelmäßig Demonstrationen. Nach dem Regierungswechsel im Herbst 2015 begannen liberalkonservativ, liberal und links eingestellte Wähler, die sich bislang selten in dieser Form engagiert hatten, auf die Straße zu gehen. Es entstand das Komitee zur Verteidigung der Demokratie (Komitet Obrony Demokracji-KOD), das seit Dezember 2015 in Kooperation mit den Oppositionsparteien Antiregierungsmärsche und -kundgebungen organisiert. Die Demonstrationen und die Ansammlung von Zelten vor dem Regierungssitz in Warschau dienen der Mobilisierung der Wähler und einer eigenen Politik der Identitätsstiftung. Die Verlagerung der politischen Aktivität auf die Straße resultiert gleichermaßen aus starken gesellschaftlichen Emotionen wie aus der Schwäche der parlamentarischen Opposition sowie des Parlaments und seiner Ausschüsse als Bühne der sachbezogenen Auseinandersetzung der Opposition mit der Regierung. Die Strategien der Oppositionsparteien ergeben sich des Weiteren aus der Analyse der Erfahrungen rechter Gruppierungen, die während der Regierungszeit der PO Aktionsformen sozialer Bewegungen nutzten, indem sie ein Netz gesellschaftlicher Organisationen um sich herum aufbauten und Märsche an Jahrestagen mit Symbolkraft organisierten.

$\mathrm{N}$ ach den Parlamentswahlen im Herbst 2015 und der Regierungsbildung durch PiS ist in Polen eine weitere Etappe der Institutionalisierung von Protest und verschiedener Arten von Demonstrationen als eine Form von politischer Kommunikation zu beobachten. Bei anhaltend hoher gesellschaftlicher Unterstützung für PiS (knapp unter 40 Prozent) und der Ritualisierung der Beratungen des Sejm wurden Proteste auf der Straße zu einer Möglichkeit für die Opposition, Widerspruch gegenüber der Regierung kundzutun und Wähler zu mobilisieren. Nach der Regierungsbildung im November 2015 gründete sich das Komitee zur Verteidigung der Demokratie, eine PiS gegenüber kritisch eingestellte Initiative. Mit der Zeit wurde $K O D$ zur Plattform oppositioneller Auftritte gegen verschiedene Aktivitäten und Programme der Regierung, insbesondere im Zusammenhang mit der Novelle des Gesetzes über das Verfassungstribunal und der Anerkennung seiner Urteilssprüche. Die parlamentarische Opposition, vor allem die führenden Köpfe der PO, von Die Moderne (Nowoczesna) und der Polnischen Bauernpartei (Polskie Stronnictwo Ludowe - PSL), schloss sich den Protesten von $K O D$ an. Seitdem werden die Protestmanifestationen unter dem Namen von $K O D$ organisiert, mit Beteiligung der untereinander rivalisierenden Oppositionsparteien, die die Aktionen teilweise aus ihrem Budget mitfinanzieren. So führt $K O D$ die in manchen gesellschaftlichen Gruppen starken Antiregierungsemotionen an.

\section{Die Institutionalisierung des Protests und die Entstehung von KOD}

Die Gewerkschaften, früher die wichtigsten Organisatoren von Protesten, dominieren die Landschaft der Pro- testpolitik in Polen nicht mehr. Die Transformation des Wirtschaftssystems und Lohnforderungen sind nicht mehr der wichtigste Rahmen und Bezugspunkt für Proteste. Gleichzeitig wurden Demonstrationszüge in den letzten Jahren so weit institutionalisiert, d. h. populär und wiederholt eingesetzt und als normale, akzeptable Form politischen Ausdrucks betrachtet, dass sich weitere, politische und ideologische Milieus bemühen, eben in dieser Form die Empörung und die Unterstützung ihrer Anhänger zu organisieren.

In den ersten sechs Monaten der Regierungszeit von PiS kam es zu einer Institutionalisierung des Protests. $\mathrm{Zu}$ beobachten sind unterschiedliche Protestformen in ihrer Wiederholbarkeit; Demonstrationen finden nicht nur in Warschau statt. Feststellbar ist auch, dass unter den Angehörigen der großstädtischen Mittelschicht, die bislang eher ungern auf die Straße gingen, das Gefühl wächst, dass ein Demonstrationszug durch die Stadt oder auch eine Zeltstadt vor dem Sitz der Ministerpräsidentin in Warschau etwas "Normales" ist. Das, was bisher Praxis der jungen Teilnehmer der »Märsche der Unabhängigkeit« oder der Mitglieder der Klubs der Gazeta Polska war, die in Zeiten der PO-Regierung PiS unterstützten, ist jetzt auch für die 50- und 60-Jährigen "normal" geworden, die negativ gegenüber der aktuellen, rechten Regierung eingestellt sind und nun $K O D$ Plakate tragen.

Das Komitee zur Verteidigung der Demokratie entstand im November 2015 als eine Facebook-Aktion. Der Name knüpft an das 1976 entstandene Komitee zur Verteidigung der Arbeiter (Komitet Obrony Robotników$K O R$ ) an und signalisiert, dass die Organisatoren von 
$K O D$ die Bedrohungen für die Freiheit im kommunistischen Polen mit der Gegenwart parallelisieren, wobei PiS eine ähnliche Gefahr für die Demokratie und Freiheit darstelle. Zum Einsatz kam auch wieder ein Element aus dem Stromschaltkreis, der Widerstand, der in den Jahren des Kriegsrechts (1981-1983) am Revers als Symbol des Widerstands gegen die Machthaber getragen wurde. Der Gründer von $K O D$, aus dem eine ganze Bewegung entstand, war Mateusz Kijowski, ein Informatiker, der sich früher u. a. in der Bewegung für die Rechte von Vätern engagiert hatte. Von Beginn an wurde die These von der falschen Richtung, in die die Veränderungen in Polen gehen, und von dem antidemokratischen und sogar totalitären Charakter von PiS deutlich formuliert: "Die Demokratie ist stark gefährdet, manche sagen, dass ein Staatsstreich stattgefunden habe und sich der Totalitarismus nähere. Deshalb wollen wir die Demokratie verteidigen. Wir sprechen uns für keine politische Partei aus, wir kämpfen weder um die Macht noch gegen konkrete Menschen «, sagte Kijowski im November 2015 der Tageszeitung Gazeta Wyborcza. ${ }^{1}$ Im Manifest der entstehenden Bewegung schreiben die Autoren mit Nachdruck von einer "autoritären Regierung « und der Perspektive eines "totalitären Polen«.

Im Herbst 2015 war es zweifelhaft, ob sich die Aktivierung in den sozialen Medien in eine echte Mobilisierung außerhalb des Smartphones oder des Computers wandeln würde. Es zeigte sich jedoch, dass die - wenn auch allzu emphatisch verfassten - Worte über die totalitäre Bedrohung auf fruchtbaren Boden fielen. Ein wichtiges Moment für die Mobilisierung und das organisierte Anwachsen der Bewegung war der Streit um das Verfassungstribunal, der sich zunächst im Sejm abspielte. PiS unternahm den Versuch, sich das Verfassungstribunal anzueignen und leitete eine wirksame Lähmung des Gerichts ein. Die Mehrheit im Sejm und die Regierung nutzten ihre Position und das Regelwerk des Sejm dazu, vor dem Hintergrund von Regelverstößen der parlamentarischen Mehrheit des letzten Sejm bei der Richterwahl ihre Forderungen nach einer Änderung des Wahlmodus von Verfassungsrichtern durchzubringen und eine tatsächliche Debatte bzw. die Befragung ihrer Kandidaten für das Richteramt in der Sitzung des parlamentarischen Rechtsausschusses zu unterbinden. Ähnlich war in den vergangenen Wahlperioden die $P O$ zusammen mit der PSL vorgegangen. Fachliche Argumente waren im Grunde nicht diskutiert worden und die Abgeordneten hatten sich eher an die Ratschläge der Regierung und der Fraktionsvorstände gehalten. Auf diese Weise tru-

1 M. Orłowski: Powstał społeczny ruch Komitet Obrony Demokracji. Ma już ponad 20 tysięcy zwolenników. Gazeta Wyborcza, 21.11.2015. gen das "geschlossene System" des Sejm und die Ritualisierung des Gesetzgebungsprozesse zusätzlich dazu bei, die Konflikte auf die Straße zu verlagern. ${ }^{2}$

Im November 2015 schrieb KOD einen offenen Brief, in dem es Präsident Andrzej Duda aufrief, drei gesetzmäßig gewählte Richter des Verfassungstribunals zu vereidigen, und organisierte am 12. Dezember eine Protestkundgebung vor dem Sitz des Gerichts, an der über 20.000 Demonstranten und Oppositionsführer teilnahmen. Parallel dazu fanden bereits kleinere Demonstrationen in einigen anderen Städten statt. Nimmt man die Proteste gegen die Verschärfung des Abtreibungsgesetztes und die pro-life Gegendemonstrationen, die feministischen Manifestationen, die Parade für Gleichheit sowie die Proteste von bestimmten Berufsgruppen wie Krankenschwestern und Zollbeamte zusammen, dann haben sich zwischen Dezember 2015 und Juni 2016 praktisch jede Woche Menschen unter verschiedenen Slogans auf der Straße versammelt. Hinzu kommt die ständige Demonstration in Form einer Reihe von Zelten, die gegenüber dem Sitz der Ministerpräsidentin aufgebaut worden sind. Diese neue Protestform initiierte die linke außerparlamentarische Oppositionspartei Gemeinsam (Razem), die auch die Idee hatte, das Urteil des Verfassungstribunals auf das Gebäude zu projizieren. Am 17. März 2016 begann $K O D$ an diesem Ort eine unbefristete Kundgebung, indem es eine Tafel aufstellte, auf der die Tage gezählt werden, die seit dem Urteilsspruch des Verfassungstribunals zur Gesetzesnovelle über das Tribunal vergangen sind. Seitdem sind die Zelte vor der Kanzlei der Ministerpräsidentin das Zentrum der Protestpolitik und gewissermaßen der Hauptsitz von $K O D$ geworden und werden hier auch Beitrittserklärungen entgegengenommen. Dieser Ort begann auch andere Protestierende anzuziehen, darunter auch diejenigen, die infolge interner Konflikte in der Bewegung eine eigene Mini-Organisation gegründet haben. Einer der Anführer aus der Anfangsphase, der nach Streitigkeiten und Vorwürfen zu Kijowskis Führungsstil und der mangelnden Demokratie innerhalb der Bewegung aus $K O D$ ausgetreten war, trat unter einem eigenen Schild - Komitee zur Verteidigung der Demokratie gegen PiS (KOD przeciw PiS - KOD PP) - in einem eigenen Zelt in einen Hungerstreik.

Der Moment, ab dem man davon sprechen kann, dass sich die Proteste der Opposition stabilisiert und institutionalisiert haben, war die große Demonstration unter dem Motto »Wir sind und bleiben in Europa« am 7. Mai in Warschau. An ihr nahmen zirka 60.000 Per-

2 Zur Ritualisierung des Gesetzgebungsprozesse in Sejm siehe Agnieszka Dudzińska: System zamknięty. Socjologiczna analiza procesu legislacyjnego. Warszawa 2015. 
sonen teil, die auf den zentral gelegenen Piłsudski-Platz zogen, um dort die Anführer von KOD und den Oppositionsparteien zu hören. Der wichtigste Organisator dieses Protestmarsches, der viel Energie und Geld in die Bekanntmachung der Veranstaltung auf Billboards und in die Transportmöglichkeiten für die Teilnehmer gesteckt hat und das eigene Logo deutlich platzierte, war die $P O$, die die Veranstaltung als Möglichkeit betrachtete, wieder Auftrieb zu bekommen. Dennoch wurde die Demonstration in der öffentlichen Debatte eher als eine von $K O D$ wahrgenommen.

Die Demonstration enthüllte eine Reihe von Interessenskonflikten zwischen den verschiedenen Akteuren der oppositionellen Protestplattform; gleichzeitig schloss sie auch die Anfangsphase der Stabilisierung der Protestszene ein halbes Jahr nach den von PiS gewonnenen Parlamentswahlen ab.

\section{Die Logik sozialer Bewegungen und politischer Parteien}

Die Gruppierungen von liberalkonservativ bis links hatten die Gewissheit erlangt, dass sie unter dem Schild von $K O D$ in der Lage sind, eine gewisse gesellschaftliche Unterstützung auf den Straßen zu mobilisieren; sie waren aber auch zu der Erkenntnis gekommen, dass sich diese nicht in Form steigender Umfragewerte auswirken wird. Ebenso wenig ist eine sinkende Unterstützung für PiS festzustellen, die mit dem Familienförderprogramm "500+« und der angekündigten Auflegung eines Programms für günstigen Wohnraum positiv bewertete Sozialreformen initiiert hat.

Gleichzeitig fanden auf dem linken Flügel der politischen Bühne Veränderungen und Versuche statt, eine optimale Position vor den Parlamentswahlen in dreieinhalb Jahren zu besetzen. KOD rief die Koalition Freiheit, Gleichheit, Demokratie (Wolność Równość Demokracja) ins Leben, der eine Reihe kleiner linker und liberaler Gruppierungen beitrat, die gegenwärtig nicht im Parlament vertreten sind (darunter auch Vorsitzende der postkommunistischen Linken), sowie auch die PSL und Die Moderne aus den Reihen der Parlamentsparteien. Nicht beigetreten ist allerdings die größte Oppositionspartei, die $P O$. Kijowski machte deutlich: »KOD ist nicht Mitglied dieser Koalition, wir sind ihr Patron, eine Verständigungsplattform für diese Parteien. [...] Wir sind nur Moderator. [...] Wir sind eine zivilgesellschaftliche Bewegung und betreiben keine Parteipolitik. $\aleph^{3}$ Allerdings kommt es immer wieder zu Mutmaßungen, ob KOD nicht doch irgendwann eine politische Partei wird und in den kommenden Parlamentswahlen

3 R. Grochal, S. Klauziński: Marsz, marsz, Kijowski. Gazeta Wyborcza, 11.05.2016. selbst antreten wird. Problematisch ist auch das Verhältnis zwischen der $P O$ und $K O D$.

Dass die Logik einer sozialen Bewegung mit der Taktik einer politischen Partei zusammenstoßen kann, macht die Geschichte des Bürger-Gesetzesprojektes zum Verfassungstribunal deutlich, das $K O D$ in den Sejm eingebracht hat und dem über 100.000 Bürger zugestimmt hatten. PiS hat dieses Projekt nicht abgewiesen, sondern an die parlamentarischen Ausschüssen weitergeleitet, was von den Oppositionsparteien und den Anführern von $K O D$ als Vorwand interpretiert wurde, um eigentlich das Gesetzesprojekt von PiS umzusetzen. Die Moderne und die PO stimmten nach Absprache mit $K O D$ schließlich gegen die Annahme des Projekts, um kein Prozedere zu legitimieren, in dem PiS die dominierende Position innehat. Sie argumentierten dahin gehend, dass zunächst alle gewählten Richter des Verfassungstribunals vereidigt und seine Urteile veröffentlicht werden müssten. Dabei verwickelte sich $K O D$ in ein schwer zu vermittelndes Spiel: Nun muss das Komitee seinen Anhängern erklären, warum es die weiteren Arbeiten an dem von ihnen in den Sejm eingebrachten Gesetzesprojekt nicht will. Hier lassen sich parteipolitische Interessen beobachten, in die sowohl KOD als auch sein Projekt hineingezogen worden sind. Anfang Juni gaben Kijowski und Grzegorz Schetyna, die Chefs von $K O D$ und der $P O$, sogar eine eigene Pressekonferenz, um dann nur so viel zu erklären, dass die Zusammenarbeit beider Gruppierungen sehr gut verlaufe und beide den "Willen zur Zusammenarbeit, Offenheit und Sympathie bekunden«. Natürlich organisiert man keine solche Pressekonferenz, wenn die Zusammenarbeit tatsächlich konfliktfrei verläuft. Sie war eher eine Bestätigung der Reibungen im oppositionellen Milieu.

\section{Ressourcen und Anhänger gewinnen}

$K O D$ funktioniert als Verein, der von zirka 20 Gründungsmitgliedern ins Leben gerufen wurde und sich aktuell bemüht, so viele eingetragene Mitglieder wie möglich zu gewinnen. Dies soll u. a. dazu dienen, den Kreis der ständigen Mitarbeiter zu erweitern und die finanziellen Mittel zu stabilisieren. Innerhalb von fünf Monaten, bis Ende April, hat $K O D$ zirka eine Million Zloty gesammelt, wobei über die Hälfte auf den Demonstrationen in Spendendosen gegeben wurde. ${ }^{4}$ Während der großen Veranstaltung in Warschau konnte $K O D$ mit Hilfe von 70 Freiwilligen zirka 180.000 Zloty sammeln. Andere Quellen sind online-crowdfunding oder individuelle Überweisungen von Anhängern. Mitglieder des Vereins leisten einen monatlichen Beitrag von fünf oder zehn Zloty und even-

4 Informacja Komitetu Społecznego: »Komitet Obrony Demokracji« z 18.05.2016, <www.ruchkod.pl> 
tuell eine zusätzliche Spende. Darüber hinaus rief $K O D$ auch öffentlich zur Finanzierung seiner Medien auf, das heißt einer Internetzeitung sowie eines künftigen Internetradios. Außerdem gibt es einige Zeitungen, die von Aktivisten erstellt und während der Demonstrationen oder auf dem Posten vor der Kanzlei der Ministerpräsidentin verteilt werden. All dies zeigt eine recht deutliche Effektivität und eine zunehmende Organisiertheit der Bewegung.

Bedeutende Ressourcen steuern auch die politischen Parteien bei, die einerseits den Namen KOD als aussagekräftiger und glaubwürdiger als ihren eigenen Parteinamen nutzen, aber andererseits die Proteste auch als die ihren sowie auch als ihren Erfolg darstellen wollen. So war es beispielsweise bei der Organisation der Demonstration im Mai. Die $P O$ mietete große Reklametafeln und Billboards, auf denen die Demonstration angekündigt wurde, sie zahlte für die Bühne und brachte einige Tausend Anhänger mit ihren Bussen zur Demonstration.

Die Ressourcen sind nicht nur materieller Art. In seiner Anfangsphase des organisierten Wachstums sucht KOD vor allem nach Legitimation. Diesem dienten u. a. die Besuche im Westen und die Treffen mit politischem Führungspersonal und Vertretern der Eliten, so zum Beispiel der Besuch einer Delegation von KOD in den USA und das Treffen mit Freedom House oder die Reise nach Brüssel und Treffen mit den Präsidenten bzw. dem Fraktionsvorsitzenden Donald Tusk, Martin Schulz, Frans Timmermans und Guy Verhofstadt. Ähnliche Bedeutung hatten die Bemühungen um den Europäischen Bürgerpreis des Europäischen Parlaments und die Bestimmung von Władysław Bartoszewski zum Patron der KOD-Bewegung.

Auch weitere politische und gesellschaftliche Unterstützung kann $K O D$ Glaubwürdigkeit oder Legitimierung bringen. Hier ist u. a. die Stadt Warschau ein wertvoller Verbündeter (die Stadtpräsidentin von Warschau ist gleichzeitig stellvertretende $P O$-Vorsitzende). Freilich gibt sie regelmäßig eine überhöhte Schätzung der Teilnehmerzahlen bei Demonstrationen von $K O D$ bekannt. Beispielsweise gab die Stadtverwaltung für die Demonstration am 7. Mai unter dem Motto "Wir sind und bleiben in Europa« die Zahl von 240.000 Teilnehmern an, gegenüber zirka 60.000 tatsächlichen Demonstranten, was an sich schon eine imponierende Zahl ist. Auf diese Weise erhielten die Organisatoren eine von ihnen als wertvoll angesehene symbolische Unterstützung. Westliche Medien, darunter auch deutsche, nahmen dies auf, indem sie von "Hunderttausenden Demonstranten gegen die Regierung in Warschau« schrieben. Einer der wichtigsten Kommentatoren der Gazeta Wyborcza, Marek Beylin, schrieb: "Wenn eine Viertelmillion Protestierender auf die Straße geht, haben alle Machthaber vor Angst die Hosen voll. Für PiS aber ist die Situation besonders bitter. Eine so mächtige gesellschaft- liche Mobilisierung zeigt, dass [...] der gesellschaftliche Widerstand nicht nur nicht erlöscht, sondern, im Gegenteil, sich noch verstärken wird. ${ }^{5}$

Diese publizistische Emphase zeigt, dass die Gazeta Wyborcza ein weiterer wertvoller Verbündeter von $K O D$ ist, dergestalt, dass sie sich vollständig mit der Botschaft der Demonstrationen identifiziert und die Proteste befürwortet. Die Kommentare sind im Tenor des "wir» protestieren verfasst, die Zeitung gibt umfangreiche Informationen über die Demonstrationen und macht für sie Werbung. Journalisten der Gazeta Wyborcza nahmen als Redner an Kundgebungen zu den öffentlichen Medien teil, ihr Herausgeber Adam Michnik stellte den bereits erwähnten Zähler vor dem Sitz der Ministerpräsidentin auf und besuchte dort die Zeltansammlung von $K O D$. Auch veröffentlichte die größte liberale Tageszeitung den Ratgeber "Was tun, wenn sie Dich schnappen", der ähnlichen Ratgebern aus der Zeit der Volksrepublik nachempfunden ist. In einem Redaktionskommentar schrieb Chefredakteur Jacek Kurski am 4. Juni: »Diese zivilgesellschaftliche Bewegung lässt sich weder ersticken noch kaufen noch verschrecken. PiS muss sich fürchten. ${ }^{6}$ Die Zeitung stellt die starke Antiregierungshaltung der Bewegung heraus und befleißigt sich einer entsprechenden Rhetorik, um KOD zu charakterisieren, auch wenn die Aussagen der führenden Aktivisten selbst eher neutral sind und auf einen nicht näher bestimmten »Aufbau einer Zivilgesellschaft« abstellen.

\section{Der Rahmen der Proteste}

Zweifellos aber beschreibt der Kommentar des Publizisten die Emotionen der Protestteilnehmer unter dem Aspekt zutreffend, dass es sich um starke Anti-PiS Emotionen handelt, das heißt negative Emotionen gegenüber der aktuellen Regierung und der sie stellenden Partei, weniger jedoch Emotionen im Hinblick auf ein kohärentes Panorama von Ansichten oder von politischen Reformen. "Jetzt hatten wir den Europatag. Anschließend kommt der 4. Juni. Allgemein gilt, wenn wir alle fünf bis sechs Wochen etwas organisieren, dann ist es gut. Die Menschen brauchen das. Nach zwei Wochen fragen sie, wann die nächste Demonstration stattfinden wird. In ihnen wächst die Empörung und das Bedürfnis sich zu treffen, gemeinsam die Unzufriedenheit zu zeigen«, sagte Kijowski über die Häufigkeit der Demonstrationen in einem Interview für die Gazeta Wyborcza. Diese Aussage verdeutlicht den Mechanismus, die Proteste aufrechtzuerhalten, sowie den Mechanismus des Drucks von Seiten der Sympathisanten, weitere Veranstaltungen durchzu-

5 M. Beylin: Wobec takiej siły PiS jest bezradny. Gazeta Wyborcza, 08.05.2016.

6 J. Kurski: Tego obywatelskiego ruchu nie da się ani zdusić, ani kupić, ani zastraszyć. Gazeta Wyborcza, 04.06.2016. 
führen. In den Darstellungen der Teilnehmer und Sympathisanten selbst wiederholen sich Schlüsselbegriffe wie "europäische Werte« und EU, »fröhliche Gesichter« der Protestteilnehmer im Gegensatz zu den »mürrischen Demonstranten bei den Kundgebungen von PiS«, der Erfolg Polens nach 1989, der von PiS bedrohte Rechtsstaat, der antipolitische Charakter von $K O D$ (in einem gewissen Gegensatz zu den politischen Zielen der Oppositionsparteien). »Ein demokratisches Polen in der Europäischen Union, ein Polen freier und gleicher Bürger diese Werte und diese unsere Lebenseinstellungen, heute bedroht, führten uns in den Demonstrationszug", so der Kommentar von der Gazeta Wyborcza. ${ }^{7}$ Der gemeinsame Rahmen dieser Manifestationen, der die Mehrheit der Teilnehmer grundsätzlich vereint, ist allerdings vor allem die Abneigung gegenüber PiS.

\section{Die Bezugnahme auf historische Jahrestage}

Eine Methode, Identität zu stiften, sind auch die häufigen Bezugnahmen auf historische Jahrestage. Nach der Manifestation zum Jahrestag der halbfreien Wahlen am 4. Juni 1989 sind im Juni weitere Demonstrationen geplant, die sich direkt auf Proteste in der Zeit der Volksrepublik beziehen, und zwar anlässlich des Jahrestags der Ereignisse in Ursus und Radom im Juni 1976 sowie aus Anlass des 60. Jahrestages der Massenproteste im Juni 1956 in Posen (Poznań). Charakteristisch ist hier die Sprache, mit der die Veranstaltungen bekannt gemacht werden. Die Jahrestage der tragischen Ereignisse im Juni 1956 in Posen sowie im Juni 1976 in Radom sind eine Gelegenheit, die Demonstrationen von $K O D$ in die Tradition der Proteste für Freiheit in der Zeit der Volksrepublik einzuordnen und den Gegner, das heißt die PiS-Regierung, als Äquivalent zu den kommunistischen Machthabern darzustellen, die die Freiheit beschränkt hatten. Die Einladung zur Teilnahme am 40. Jahrestag der Ereignisse in Radom lautet: "Am 25. Juni 2016 lädt das Komitee zur Verteidigung der Demokratie alle um 11.00 Uhr zum Gedenkmarsch für die Helden des Juni ein, um des Kampfes für die Freiheit zu gedenken, der im Jahr 1976 begann. Erlauben wir nicht, uns die Freiheit nehmen zu lassen, für die so viele ihr ganzes Leben geopfert haben. Gedenken wir der Arbeiter, die für ihre Rechte kämpften, gedenken wir der Entstehung des $K O R$ und der $R O P C i O$, der Anfänge des erfolgreichen Kampfes für Demokratie ${ }^{8}$ (ROPCiO - Ruch Obrony Praw Człowieka i ObywatelalBewegung zur Verteidigung der Menschen-und Bürgerrechte; oppositionelle Organisation in der Volkrepublik Polen, Anm.d.Red.).

\footnotetext{
7 M. Beylin: Wobec takiej siły PiS jest bezradny, Gazeta Wyborcza, 8.05.2016:

8 <www.ruchkod.pl>
}

\section{Das Beispiel PiS und rechter Bürgernetzwerke}

Die emotionale Anknüpfung an die Phase der Volksrepublik Polen ist nichts Neues in der polnischen Protestkultur. Die von linken und liberalen Gruppierungen und Bewegungen organisierten Demonstrationen entstanden nicht aus dem Nichts. Ihre Organisatoren griffen auf Mobilisierungs- und Protestpraktiken zurück, die vorher bereits vor allem im rechten Milieu erprobt worden waren, und zwar insbesondere von der aktuell regierenden PiS wie auch der Nationalen Bewegung (Ruch Narodowy). Den extremen Rechten gelang als ersten der Versuch, Mobilisierung in Form von Demonstrationen zu initiieren. Seit dem Jahr 2010 begannen sie, ihre ideologische Strömung nicht mehr nur über die Aktivitäten einer klassischen politischen Partei oder ihre Organisation Allpolnische Jugend (Młodzież Wszechpolska) zu konsolidieren, sondern den Schwerpunkt auf den eigens berufenen Verband Marsch der Unabhängigkeit (Stowarzyszenie Marsz Niepodlegtości) und die alljährliche Demonstration am 11. November, dem Tag der Unabhängigkeit von 1918, zu legen.

Auch für PiS, damals die größte Oppositionspartei im Sejm, wurden Märsche ein wichtiges Mobilisierungsinstrument. Die jeden Monat stattfindenden Märsche anlässlich der Flugzeugkatastrophe von Smolensk (10. April 2010) zogen viele Menschen an. Im Bewusstsein des mobilisierenden Effekts solcher Aktivitäten rief PiS im Jahr 2011 einen weiteren Marsch ins Leben. Wesentlich war hier zweifellos auch die Rivalität mit dem nationalen Milieu rechts von PiS, das den Jahrestag des 11. November dominierte. So organisierte PiS im Jahr 2011 den Marsch der Unabhängigkeit und Solidarität am Jahrestag der Verhängung des Kriegsrechts am 13. Dezember 1981 unter dem Motto "Es gibt kein gerechtes Europa ohne ein unabhängiges Polen«. Das Spiegelbild dieses Marsches und dieses Mottos ist der Demonstrationszug von $K O D$, der $P O$, der PSL und von Die Moderne am 7. Mai 2016, dessen ideeller Rahmen die Betonung der Verankerung Polens in Europa und der Europäischen Union war. Auf die Demonstrationen von PiS, die der Opfer des Kriegsrechts gedenken und für PiS ein wichtiges identitätsstiftendes Element sind, antwortet $K O D$ mit Manifestationen zum Jahrestag des Juni 1956 in Posen und des Juni 1976 in Radom.

Auch andere Themen und Mottos werden aufgegriffen. Im Jahr 2014 hatte PiS zu einem Marsch »zur Verteidigung der Demokratie und Medienfreiheit« aufgerufen. Jarosław Kaczyński, PiS-Vorsitzender, hatte damals die $P O$ kritisiert, dass »die Machthaber die Gerichte unter Beteiligung des Präsidenten der Republik terrorisieren«. Paradoxerweise wurde dieses Argument von PiS vom Dezember 2014 fast genauso am selben Ort 
und in derselben Form ein Jahr später von der anderen Seite des Konflikts, also KOD und $P O$, wiederholt, wobei die Themen öffentliche Medien und Rechtsprechung in einen neuen Kontext gestellt wurden.

Der Versuch der $P O$, analog zu $P i S$ in deren Zeiten als Oppositionspartei ein Netz von kooperierenden lokalen zivilgesellschaftlichen Gruppen aufzubauen, ist eine weitere Anknüpfung an die Strategien von PiS. PiS war sich recht früh der Bedeutung eines solchen Netzes bewusst geworden und begann, um die Partei herum zivilgesellschaftliche Organisationen zu fördern. Die Klubs der Gazeta Polska, die von der PiS nahestehenden Tageszeitung Gazeta Polska gegründet wurden, sowie die »Familie« des nationalkatholischen Radio Maryja spielten hier die wichtigste Rolle. Diese konservativen und dem rechten politischen Spektrum nahe stehenden zivilgesellschaftlichen Organisationen erlaubten der Partei, schwierigere Phasen zu überstehen und ihre Anhänger zu mobilisieren.

\section{Die Bürgerklubs der PO}

In diesem Geist, allerdings von oben und zentral veranlasst, beschloss die PO ein entsprechendes Netzwerk kooperierender Kreise aufzubauen. Anfang März 2016 eröffnete sie ein Treffen der sogenannten Bürgerklubs, die eine Antwort auf Mobilisierungsprobleme der $P O$ sein sollen. Die Partei erlebt aktuell eine Krise, sehr viele Mitglieder sind nicht aktiv, die Anzahl der Beitragszahler sinkt, der Konflikt zwischen dem Flügel um den neuen Vorsitzenden Grzegorz Schetyna und dem um die ehemalige Ministerpräsidentin Ewa Kopacz dauert an. Die Umfragewerte fielen auf zirka 15 Prozent, womit die $P O$ von Die Moderne überholt wird. In dieser Situation griff die $P O$ nach der Idee der Bürgerklubs. „Die Bürgerklubs sind der Anfang einer neuen $P O$, wir wollen eine neue Etappe eröffnen - Debatten, Diskussionen über die wichtigsten Dinge«, sagte Schetyna. In der Tageszeitung Rzeczpospolita unterstrich er, dass »die Bürgerklubs als offene, unparteiliche Diskussionsorte gedacht sind«. Unparteilich, aber gleichzeitig sollen sie »der Anfang einer neuen $P O$ «sein. Der Plan sieht vor, dass sie in jedem Kreis entstehen und insgesamt zirka 400 lokale Diskussionsorte umfassen sollen, d. h. Strukturen, die die Mobilisierung der $P O$-Anhänger im jeweiligen Ort fördern und lokale gesellschaftliche Akteure zusammenführen sollen.

Auf einem Treffen der Klubs der Gazeta Polska im Mai 2015 hatte Kaczyński eindeutig über die Strategie der sozialen Bewegung gesprochen: „Ohne gesellschaftliche Unterstützung, die auch auf der Straße zum Ausdruck kommt, wird es uns nicht gelingen." Auch die aktuellen Oppositionsführer scheinen so zu denken, doch währen die Klubs der Gazeta Polska mehrheitlich von unten gewachsen sind, wurden die Bürgerklubs von oben erschaffen, als von der Partei initiiertes
Projekt zum Aufbau eines Netzwerks. Wiederum von der Basis ausgehend, bilden sich Netzwerke im Rahmen von $K O D$, und es werden wohl eher diese sein, die die Infrastruktur des liberalen Protests in den kommenden Jahren stellen werden.

\section{Fazit}

$K O D$ als eine bestimmte Form der Mobilisierung der liberal-linken Wählerschaft hat seine Position im Laufe des ersten halben Regierungsjahres von PiS stabilisiert. Der Erfolg von $K O D$ bedeutet, dass sich das ganze Segment der Aktivitäten der Opposition nach außerhalb des Sejm verlagert hat, was sich u. a. aus der Schwäche des Sejm angesichts der Dominanz von PiS in der Regierung und im Parlament, aber auch aus der Schwäche der Oppositionsparteien ergibt. Die Proteste von KOD sind "normal" geworden und ermuntern auch diejenigen, auf die Straße zu gehen, die sich bisher nicht in politischen Angelegenheiten engagiert hatten. Vereinfacht kann man sagen, dass dies die großstädtische Mittelschicht mittleren Alters betrifft. In gewisser Weise lässt sich aber auch feststellen, dass $K O D$ »die Opposition in den Schlaf wiegt«, wie Tomasz Sawczuk vom Internetmagazin Kultura Liberalna formulierte, ${ }^{9}$ und ihr die Lebenskräfte aussaugt, indem es die Energie und Emotionen der Wähler woandershin leitet. $K O D$ befreit die Oppositionsparteien davon, zu denken und tätig zu werden. Es verschlingt Energie und Emotionen, die in der Parteipolitik genutzt werden könnten. Die Oppositionsparteien ihrerseits sind schwach und rivalisieren zudem auch noch untereinander.

Ein Problem von $K O D$ ist auch eine gewisse Nähe zum Establishment (ehemalige Präsidenten, viele Künstler und Berühmtheiten unterstützen die Bewegung) und eine tendenziell protektionistische Einstellung den $P i S$ Wählern gegenüber. Maciej Kozłowski, ehemaliger Botschafter und eines der Mitglieder der Delegation von $K O D$, die nach Brüssel reiste, sagte dort: "The problem is not with PiS, it's with the people who voted for them. They are uneducated in civic values." ${ }^{10}$ Gerade diese Überzeugung, dass man das Monopol auf die zivilgesellschaftlichen Werte besitzt, und der hohe Grad an Exaltation, was auch die Bezeichnung Komitee zur Verteidigung der Demokratie widerspiegelt, sind eine Gefahr für die Protestbewegung.

Übersetzung aus dem Polnischen: Silke Plate

Informationen über den Autor finden Sie auf der nächsten Seite.

9 T. Sawczuk: KOD usypia opozycję. Kultura Liberalna Nr 382, 7.05.2016,<kulturaliberalna.pl/2016/05/07/kod-usypia-opozycje/>

10 A. Eriksson: What is KOD, the Polish pro-democracy movement?, <https://ueobserver.com/political/133698> 
Über den Autor

Mateusz Fałkowski ist Soziologe am Institut für Politische Studien der Polnischen Akademie der Wissenschaften in Warschau (Polska Akademia Nauk, Warszawa). Er ist Ko-Leiter einer Forschergruppe, die sich mit der SolidarnośćBewegung der 1980er Jahre (<http://solidarnosc.collegium.edu.pl/>) sowie zeitgenössischer Protestpolitik beschäftigt.

\section{Bewertung der politischen Situation}

Grafik 1: Wen würden Sie wählen, wenn am nächsten Sonntag Parlamentswahlen wären? (\%)

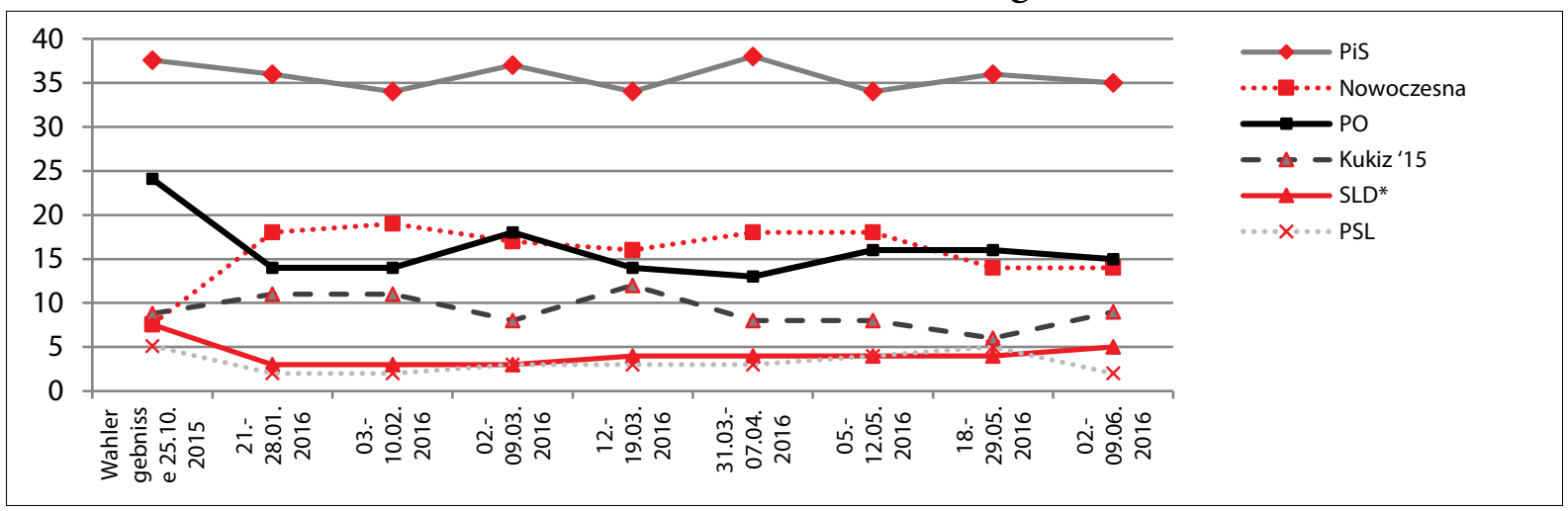

Anmerkungen und Quellen: siehe Tabelle 1.

Tabelle 1: Wen würden Sie wählen, wenn am nächsten Sonntag Parlamentswahlen wären? (\%)

\begin{tabular}{|c|c|c|c|c|c|c|c|c|c|}
\hline & $\begin{array}{c}\text { Wahl- } \\
\text { ergebnis } \\
25.10 \text {. } \\
2015\end{array}$ & $\begin{array}{c}21 .- \\
28.01 . \\
2016\end{array}$ & $\begin{array}{c}03 .- \\
10.02 . \\
2016\end{array}$ & $\begin{array}{c}02 .- \\
09.03 . \\
2016\end{array}$ & $\begin{array}{c}12 .- \\
19.03 . \\
2016\end{array}$ & $\begin{array}{c}31.03 .- \\
07.04 . \\
2016\end{array}$ & $\begin{array}{c}05 .- \\
12.05 . \\
2016\end{array}$ & $\begin{array}{c}18 .- \\
29.05 . \\
2016\end{array}$ & $\begin{array}{r}02 .- \\
09.06 . \\
2016\end{array}$ \\
\hline PiS & 37,58 & 36 & 34 & 37 & 34 & 38 & 34 & 36 & 35 \\
\hline $\mathrm{PO}$ & 24,09 & 14 & 14 & 18 & 14 & 13 & 16 & 16 & 15 \\
\hline Nowoczesna & 7,60 & 18 & 19 & 17 & 16 & 18 & 18 & 14 & 14 \\
\hline Kukiz '15 & 8,81 & 11 & 11 & 8 & 12 & 8 & 8 & 6 & 9 \\
\hline PSL & 5,13 & 2 & 2 & 3 & 3 & 3 & 4 & 5 & 2 \\
\hline SLD & $7,55^{*}$ & 3 & 3 & 3 & 4 & 4 & 4 & 4 & 5 \\
\hline Razem & 3,62 & 2 & 2 & 2 & 2 & 3 & 2 & 3 & 3 \\
\hline KORWiN & 4,76 & 4 & 3 & 2 & 6 & 2 & 3 & 3 & 3 \\
\hline andere & 0,87 & 1 & 1 & 1 & 1 & 1 & 1 & 1 & 1 \\
\hline schwer zu sagen & - & 7 & 9 & 8 & 8 & 9 & 10 & 11 & 12 \\
\hline keine Antwort & - & 1 & 1 & 1 & 1 & 1 & 0 & 1 & 0 \\
\hline
\end{tabular}

Anm.: Berücksichtigt wurden die Antworten derer, die angaben, wählen zu gehen.

* Das Ergebnis gilt für die Zjednoczona Lewica/Vereinigte Linke (= SLD + Twój Ruch + PPS + UP + Zieloni).

PiS/Prawo i Sprawiedliwość - Recht und Gerechtigkeit; PO/Platforma Obywatelska - Bürgerplattform; Nowoczesna - Die Moderne; PSL - Polskie Stronnictwo Ludowe - Polnische Bauernpartei; SLD/Sojusz Lewicy Demokratycznej - Demokratische Linksallianz; Razem - Gemeinsam; KORWiN/Koalicja Odnowy Rzeczypospolitej Wolność i Nadzieja - Koalition für die Erneuerung der Republik Freiheit und Hoffnung

Quelle: CBOS Komunikat z Badań Nr 83/2016: Preferencje partyjne w czerwcu [Parteipräferenzen im Juni]. Warszawa 06/2016. $<$ www.cbos.pl> 
Grafik 2: Das Verhältnis zur Regierung von Ministerpräsidentin ... (\%)

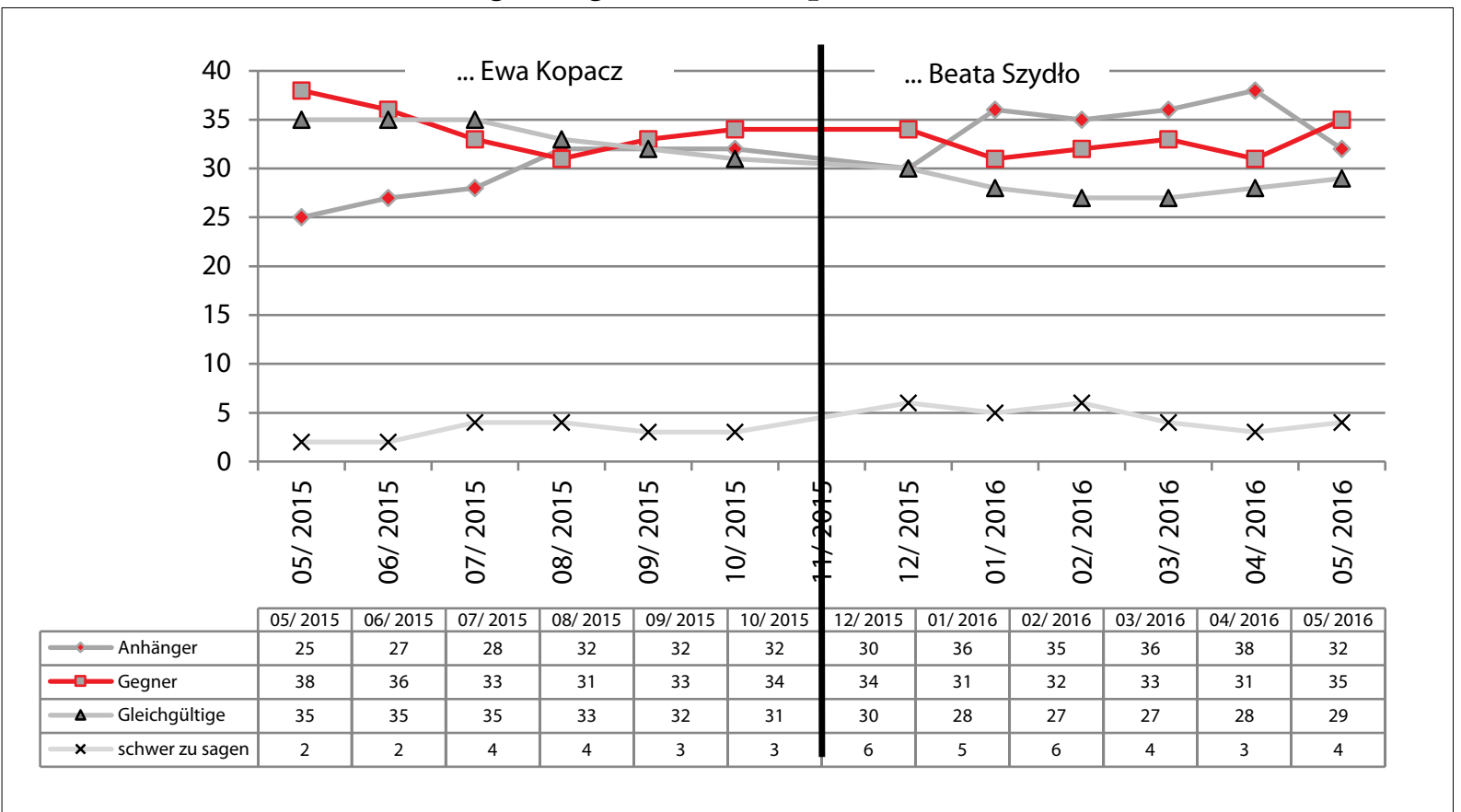

Quelle: CBOS Komunikat z Badań Nr 75/2016: Spadek notowań rządu w maju [Die Verschlechterung der Umfragewerte zur Regierung im Mai]. Warszawa 05/2016. <www.cbos.pl>

Grafik 3: Die Beurteilung der Tätigkeit des Sejm (\%)

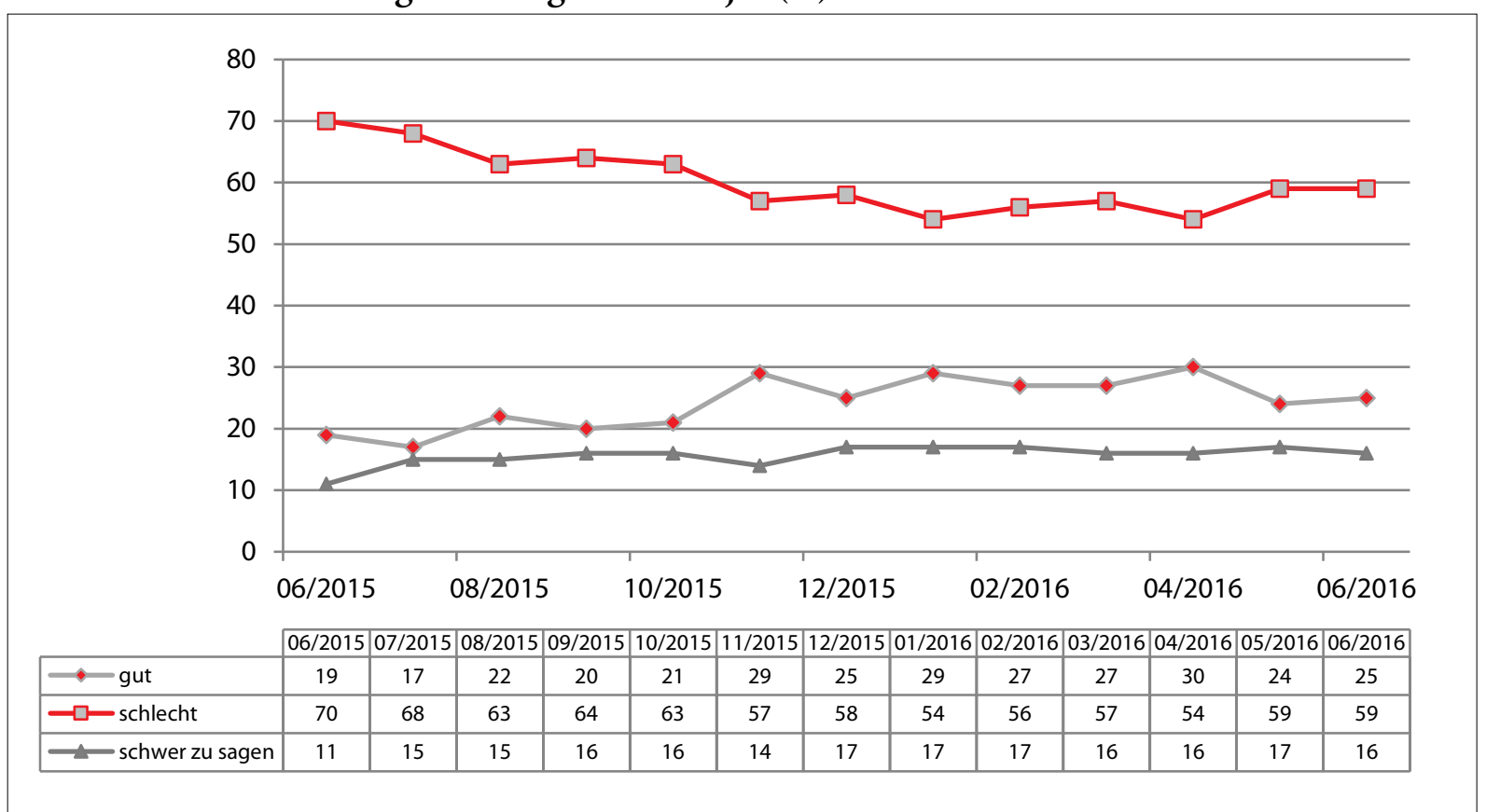

Quelle: CBOS Komunikat z Badań Nr 84/2016: Opinie o dziatalności parlamentu, prezydenta i Trybunatu Konstytucyjnego [Meinungen zur Tätigkeit des Parlaments, des Präsidenten und des Verfassungstribunals]. Warszawa 06/2016. <www.cbos.pl> 
Grafik 4: Die Beurteilung der Tätigkeit des Senats (\%)

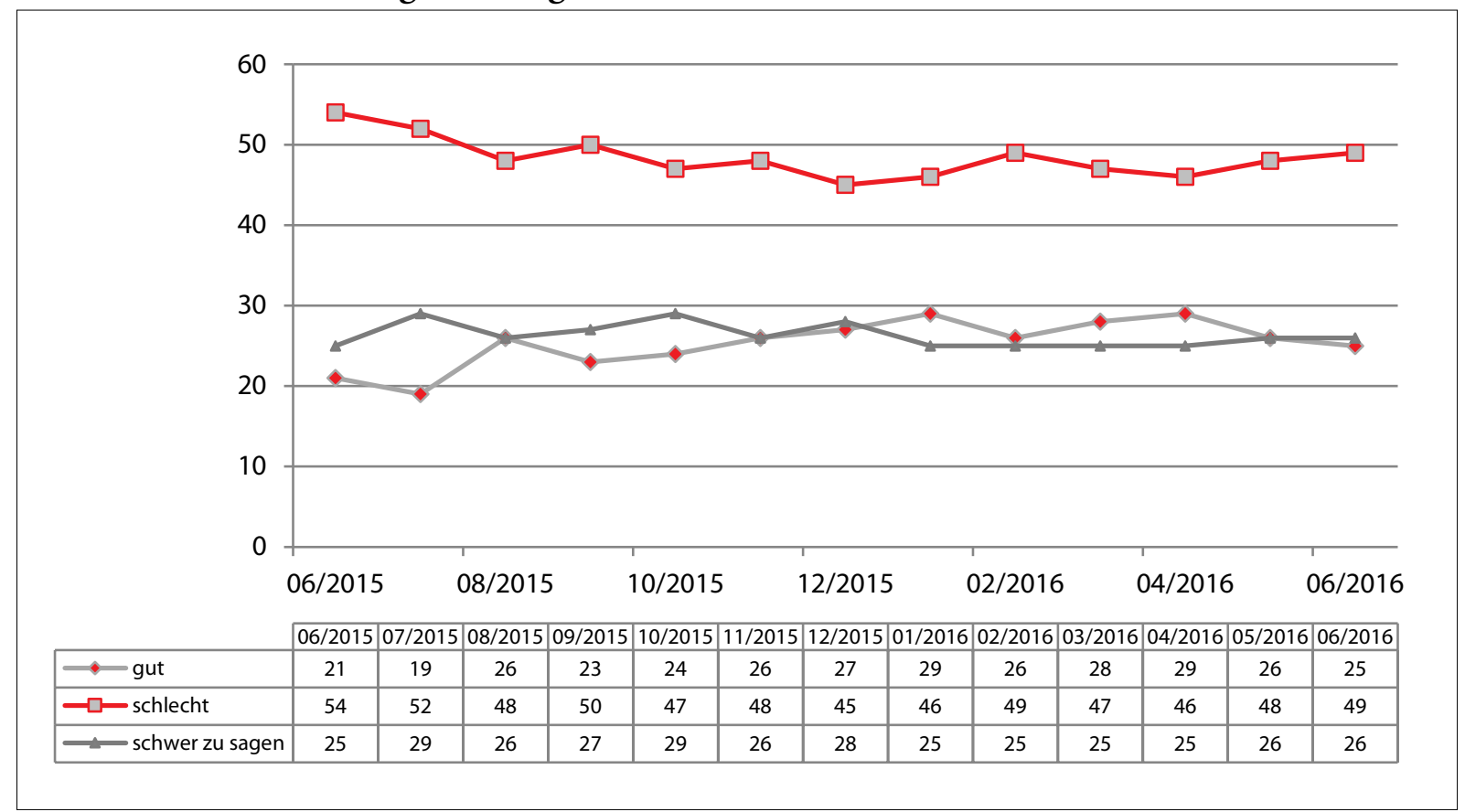

Quelle: CBOS Komunikat z Badań Nr 84/2016: Opinie o działalności parlamentu, prezydenta i Trybunału Konstytucyjnego [Meinungen zur Tätigkeit des Parlaments, des Präsidenten und des Verfassungstribunals]. Warszawa 06/2016. <www.cbos.pl>

\section{Grafik 5: Die Beurteilung der Tätigkeit des Präsidenten (\%)}

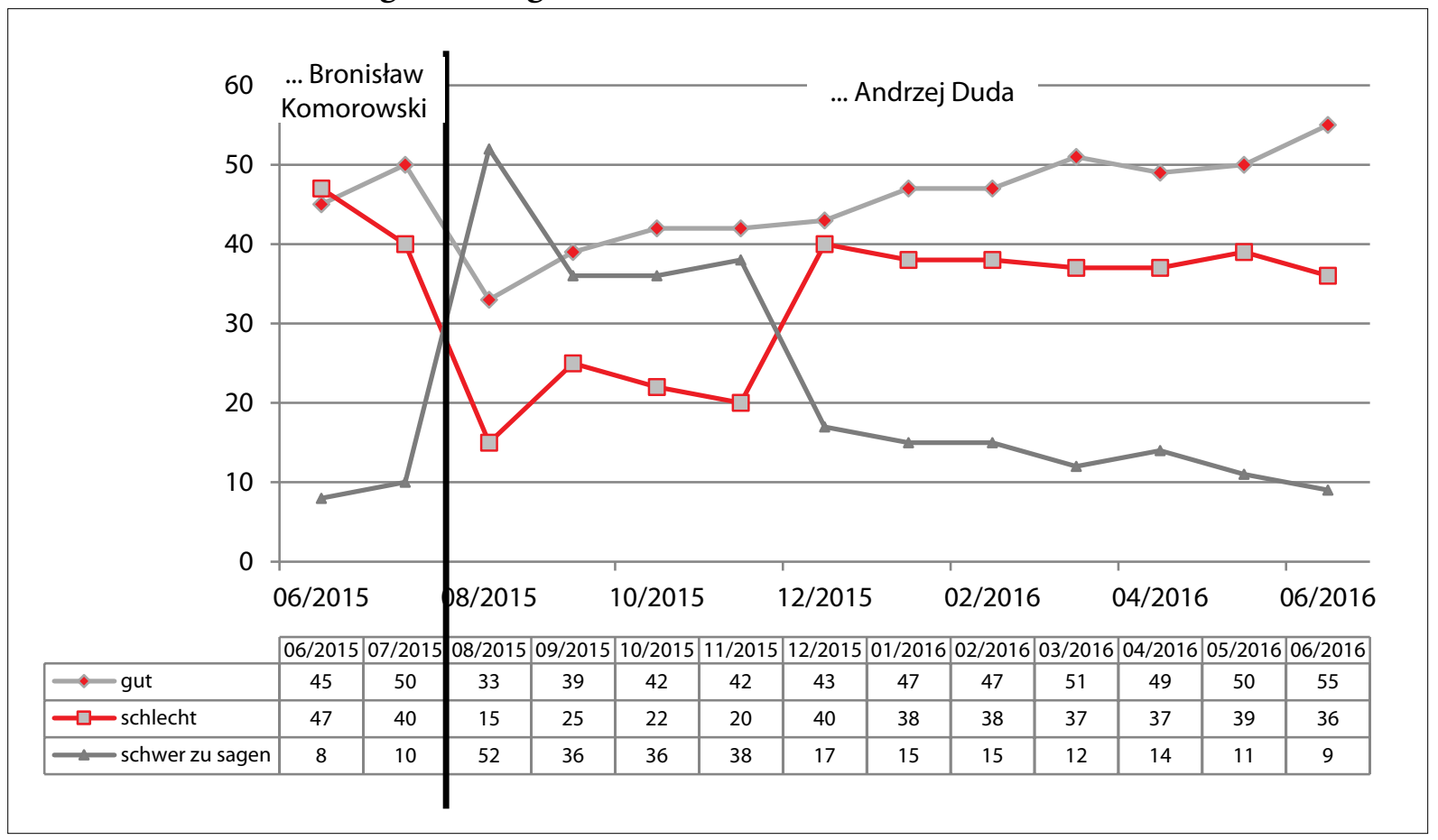

Quelle: CBOS Komunikat z Badań Nr 84/2016: Opinie o działalności parlamentu, prezydenta i Trybunału Konstytucyjnego [Meinungen zur Tätigkeit des Parlaments, des Präsidenten und des Verfassungstribunals]. Warszawa 06/2016. <www.cbos.pl> 
Grafik 6: Die Beurteilung der Tätigkeit des Präsidenten Andrzej Duda nach potentiellen Wählerschaften $(\%)$

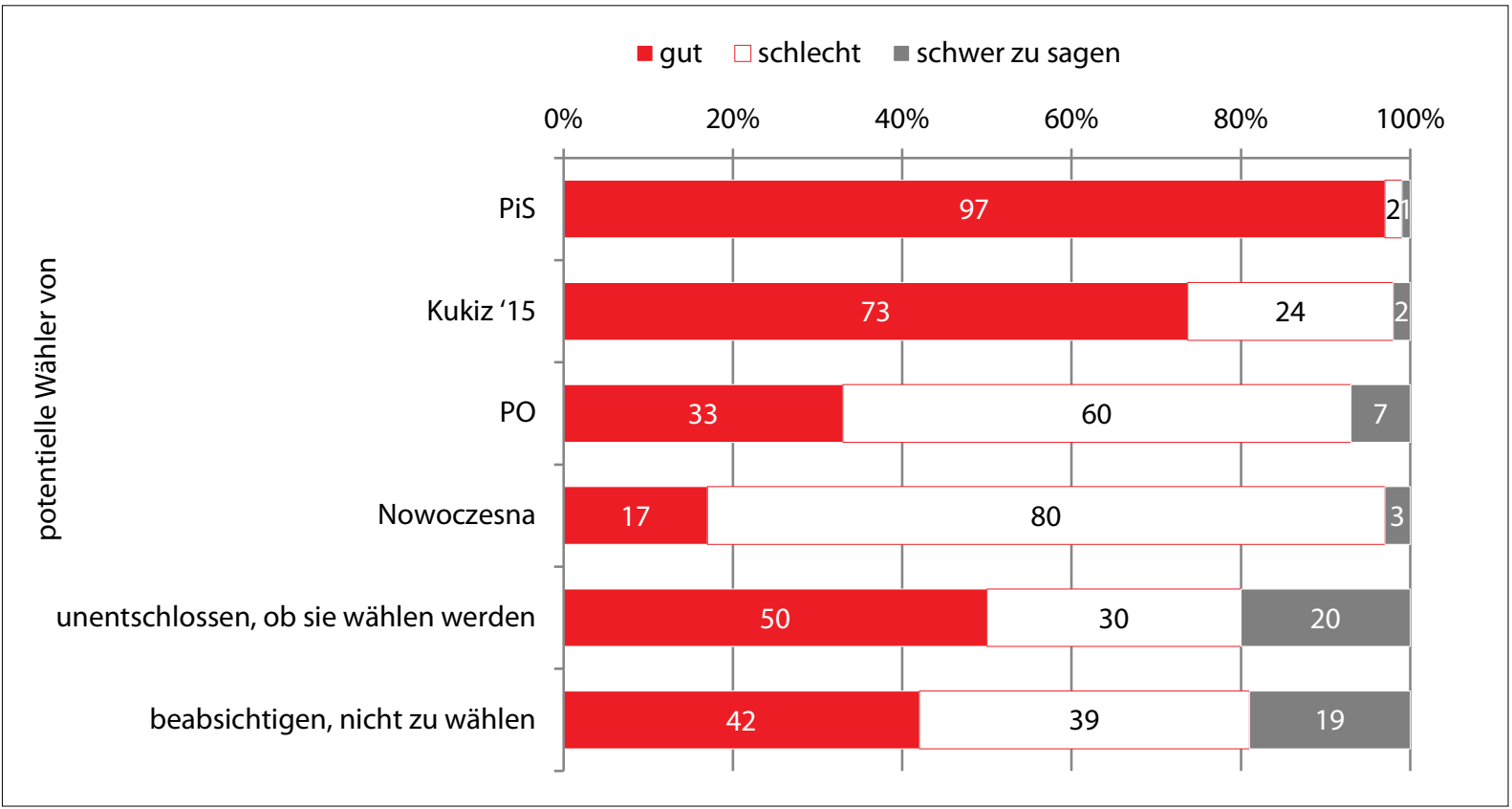

PiS/Prawo i Sprawiedliwość - Recht und Gerechtigkeit; PO/Platforma Obywatelska - Bürgerplattform; Nowoczesna - Die Moderne Quelle: CBOS Komunikat z Badań Nr 84/2016: Opinie o działalności parlamentu, prezydenta i Trybunału Konstytucyjnego [Meinungen zur Tätigkeit des Parlaments, des Präsidenten und des Verfassungstribunals]. Warszawa 06/2016. <www.cbos.pl>

\section{Grafik 7: Die Beurteilung der Tätigkeit des Verfassungstribunals (\%)}

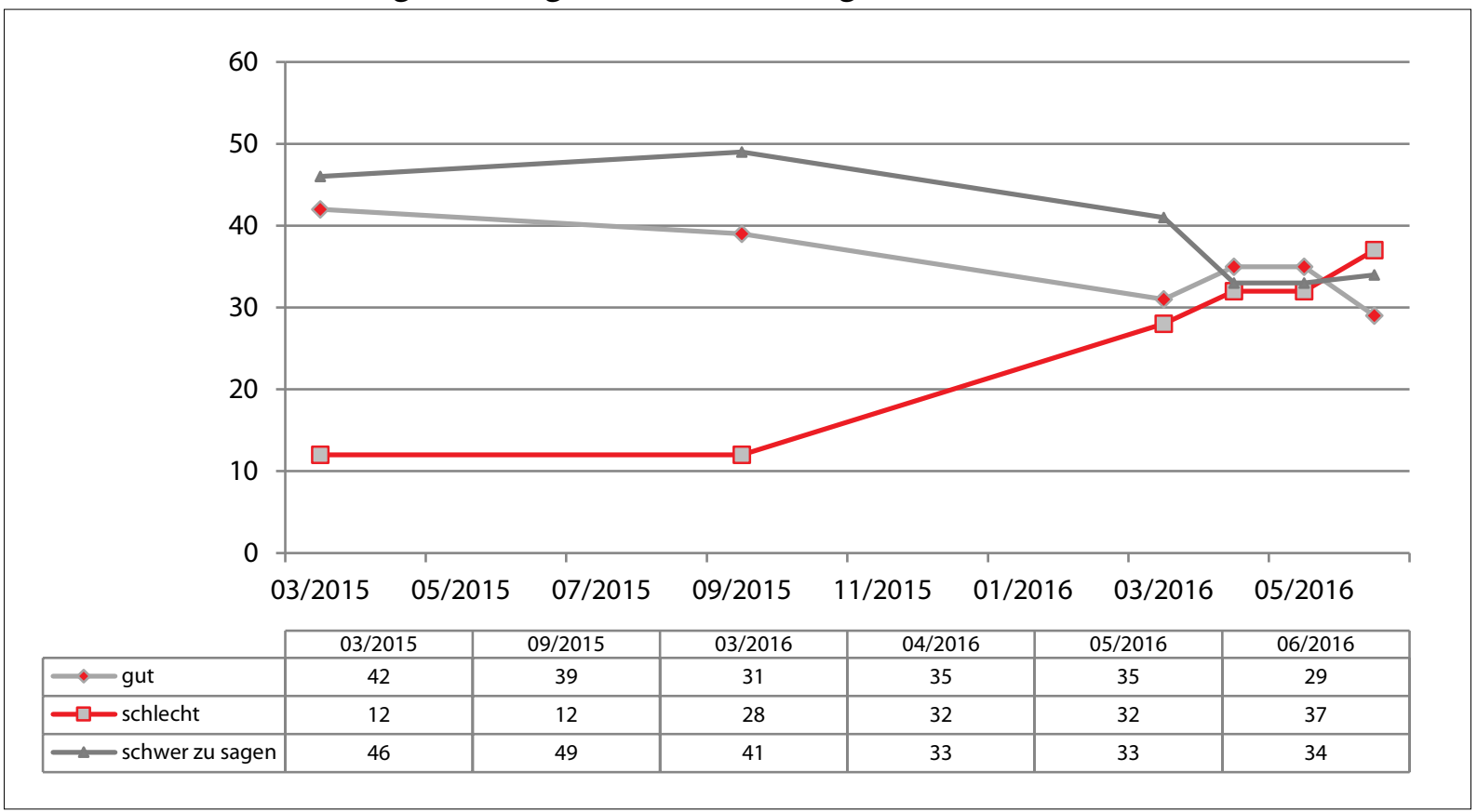

Quelle: CBOS Komunikat z Badań Nr 84/2016: Opinie o dziatalności parlamentu, prezydenta i Trybunatu Konstytucyjnego [Meinungen zur Tätigkeit des Parlaments, des Präsidenten und des Verfassungstribunals]. Warszawa 06/2016. <www.cbos.pl> 


\section{Grafik 8: Einstellungen zu Politikern, Mai 2016 (\%)}

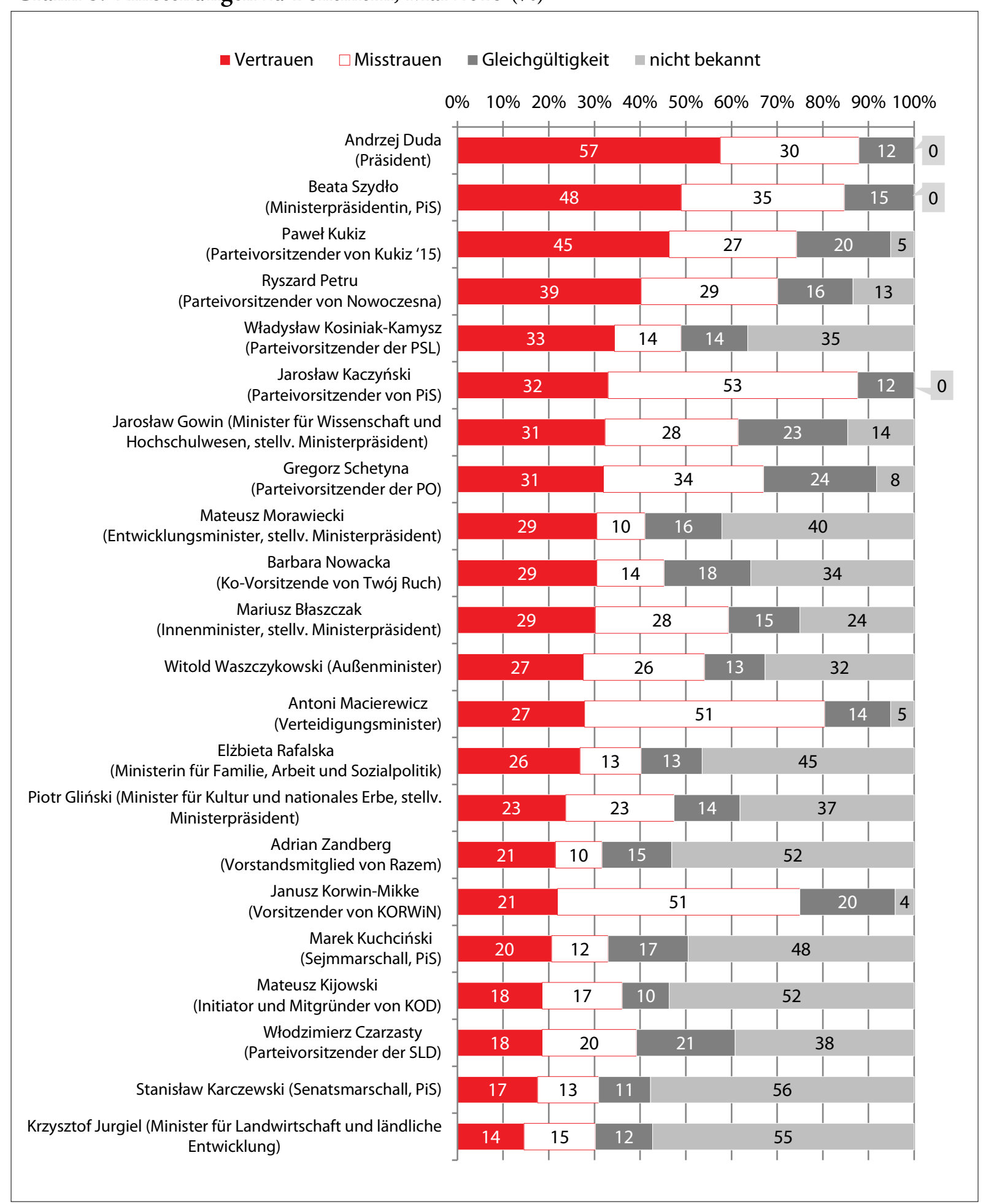

PiS/Prawo i Sprawiedliwość - Recht und Gerechtigkeit; Nowoczesna - Die Moderne; PSL/Polskie Stronnictwo Ludowe - Polnische Bauernpartei; PO/Platforma Obywatelska - Bürgerplattform; Twój Ruch - Deine Bewegung; Razem - Gemeinsam; KORWiN/Koalicja Odnowy Rzeczypospolitej Wolność i Nadzieja - Koalition für die Erneuerung der Republik Freiheit und Hoffnung; KOD/Komitet Obrony Demokracji - Komitee zur Verteidigung der Demokratie; SLD/Sojusz Lewicy Demokratycznej - Demokratische Linksallianz; Quelle: CBOS Komunikat z Badań Nr 77/2016: Zaufanie do polityków w maju [Vertrauen zu Politikern im Mai]. Warszawa 05/2016. $<$ www.cbos.pl> 


\section{Grafik 9: Einstellungen zu Politikern: Änderungen im Wert für Vertrauen von April 2016 zu Mai 2016 (\%)}

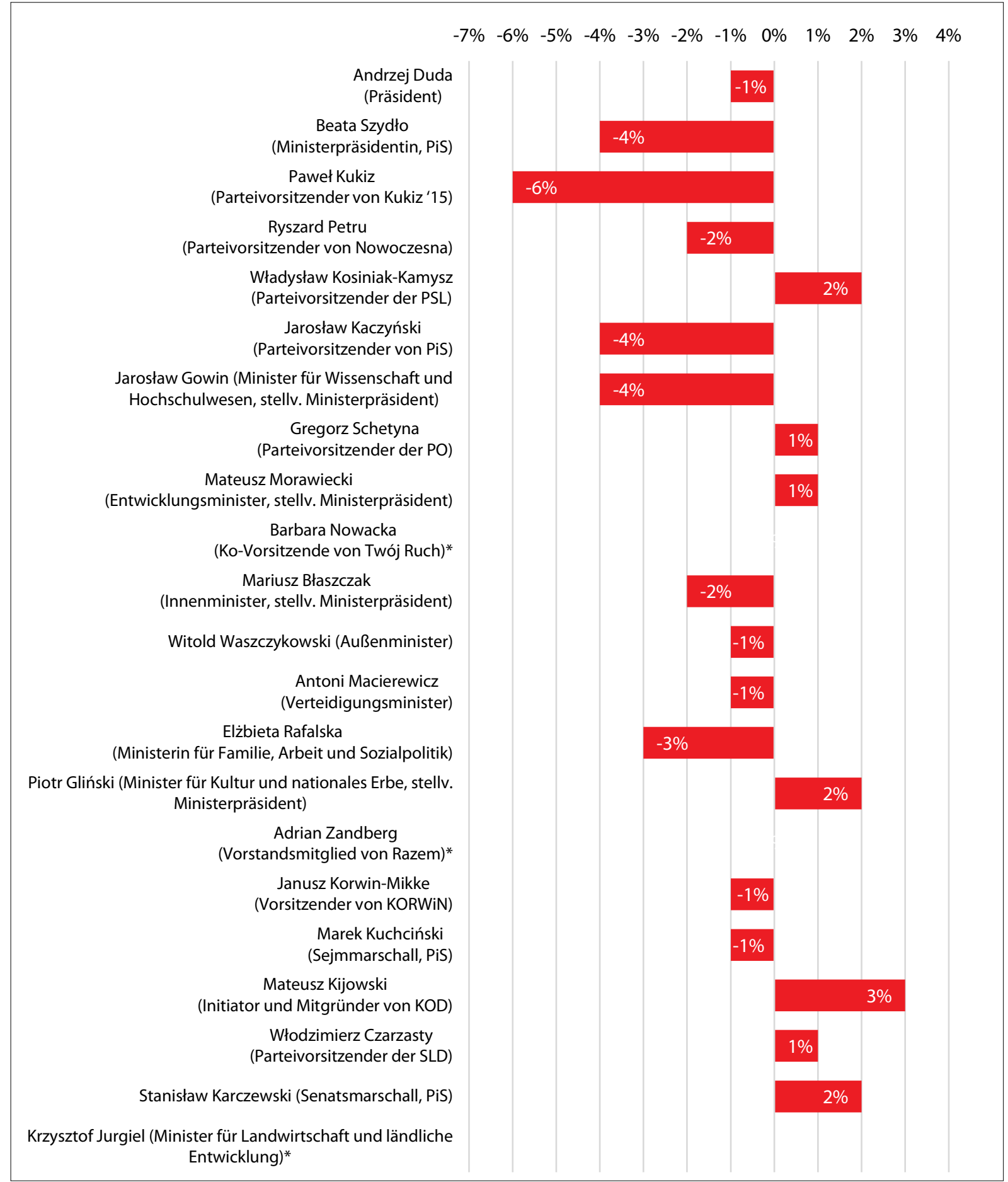

*Wurde im April 2016 nicht abgefragt.

PiS/Prawo i Sprawiedliwość - Recht und Gerechtigkeit; Nowoczesna - Die Moderne; PSL/Polskie Stronnictwo Ludowe - Polnische Bauernpartei; PO/Platforma Obywatelska - Bürgerplattform; Twój Ruch - Deine Bewegung; Razem - Gemeinsam; KORWiN/Koalicja Odnowy Rzeczypospolitej Wolność i Nadzieja - Koalition für die Erneuerung der Republik Freiheit und Hoffnung; KOD/Komitet Obrony Demokracji - Komitee zur Verteidigung der Demokratie; SLD/Sojusz Lewicy Demokratycznej - Demokratische Linksallianz; Quelle: CBOS Komunikat z Badań Nr 77/2016: Zaufanie do polityków w maju [Vertrauen zu Politikern im Mai]. Warszawa 05/2016. $<$ www.cbos.pl> 


\section{Grafik 10: Einstellungen zu Politikern: Änderungen im Wert für Misstrauen von April 2016 zu} Mai $2016(\%)$

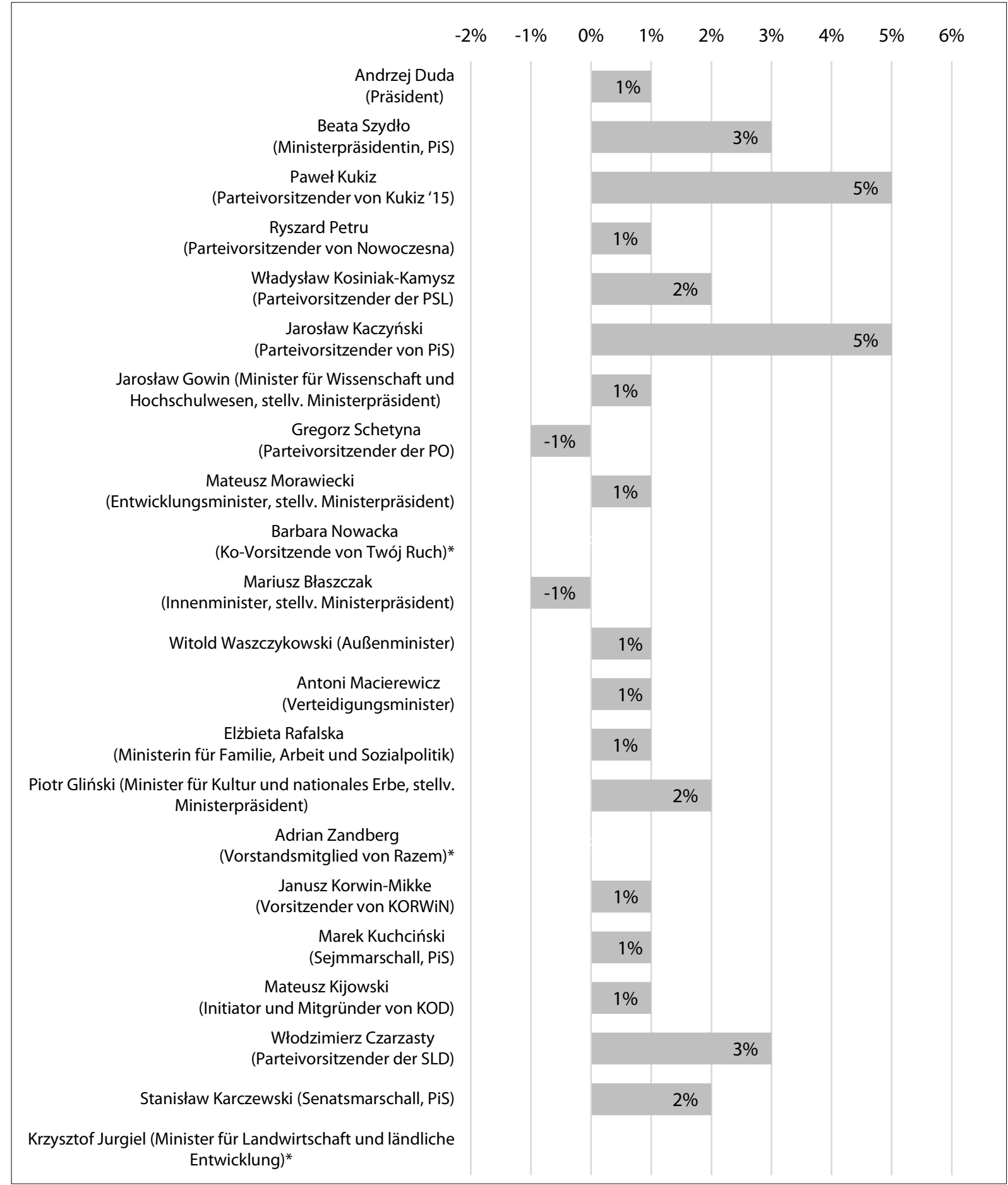

*Wurde im April 2016 nicht abgefragt.

PiS/Prawo i Sprawiedliwość - Recht und Gerechtigkeit; Nowoczesna - Die Moderne; PSL/Polskie Stronnictwo Ludowe - Polnische Bauernpartei; PO/Platforma Obywatelska - Bürgerplattform; Twój Ruch - Deine Bewegung; Razem - Gemeinsam; KORWiN/Koalicja Odnowy Rzeczypospolitej Wolność i Nadzieja - Koalition für die Erneuerung der Republik Freiheit und Hoffnung; KOD/Komitet Obrony Demokracji - Komitee zur Verteidigung der Demokratie; SLD/Sojusz Lewicy Demokratycznej - Demokratische Linksallianz; Quelle: CBOS Komunikat z Badań Nr 77/2016: Zaufanie do polityków w maju [Vertrauen zu Politikern im Mai]. Warszawa 05/2016. $<$ www.cbos.pl> 


\section{7. - 20. Juni 2016}

\begin{tabular}{|c|c|}
\hline 07.06.2016 & $\begin{array}{l}\text { In Polen beginnt das NATO-Großmanöver »Anakonda«, an dem } 31.000 \text { Soldaten teilnehmen. Neben den } \\
\text { NATO-Mitgliedern sind auch Partnerländer wie Schweden, Georgien und die Ukraine beteiligt. Ein Sprecher } \\
\text { der russischen Regierung kritisiert, dass die Übung nicht dazu beitrage, eine Atmosphäre des Vertrauens und } \\
\text { der Sicherheit zu schaffen; es bestehe ein Vertrauensdefizit im Verhältnis zum Westen. }\end{array}$ \\
\hline 08.06 .2016 & $\begin{array}{l}\text { In einer gemeinsamen Erklärung spricht sich die Visegrád-Gruppe (Polen, Tschechien, Slowakei und Ungarn) } \\
\text { auf ihrem Gipfel in Prag gegen eine Quotenregelung zur Verteilung von Immigranten in der Europäischen } \\
\text { Union bzw. gegen die Zahlung von »Strafen« aus, wenn ein Land keine Immigranten aufnimmt. Minister- } \\
\text { präsidentin Beata Szydło sagt, Polen sei, wie die anderen Länder der Region, bereit, sich außerordentlich beim } \\
\text { Schutz der EU-Außengrenzen zu engagieren und den Beitrag zur Entwicklungshilfe und zu humanitärer Hilfe } \\
\text { zu stärken. Auf dem Gipfel übernimmt Polen die Präsidentschaft der Visegrád-Gruppe von der Tschechischen } \\
\text { Republik. Der Vorsitz wechselt jährlich. }\end{array}$ \\
\hline 09.06.2016 & $\begin{array}{l}\text { Im Sejm findet die erste Lesung dreier Entwürfe zum Gesetz über das Verfassungstribunal statt. Die Entwürfe } \\
\text { wurden von Recht und Gerechtigkeit (Prawo i Sprawiedliwość - PiS), der Polnischen Bauernpartei (Polskie } \\
\text { Stronnictwo Ludowe - PSL) und der Bürgerinitiative Komitee zur Verteidigung der Demokratie (Komitet } \\
\text { Obrony Demokracji - KOD) eingebracht. }\end{array}$ \\
\hline 10.06 .2016 & $\begin{array}{l}\text { Der Sejm spricht sich für die Fortsetzung der Beratung über die drei Entwürfe zum Gesetz über das Verfas- } \\
\text { sungstribunal von Recht und Gerechtigkeit (Prawo i Sprawiedliwość - PiS), der Polnischen Bauernpartei (Pol- } \\
\text { skie Stronnictwo Ludowe - PSL) und der Bürgerbewegung Komitee zur Verteidigung der Demokratie (Komi- } \\
\text { tet Obrony Demokracji - KOD) aus. Am Vortag hat Die Moderne (Nowoczesna) einen Antrag auf Ablehnung } \\
\text { der Gesetzesprojekte von PiS und der PSL gestellt, mit der Begründung, dass diese verfassungswidrige Vor- } \\
\text { schläge enthalten würden. }\end{array}$ \\
\hline 13.06 .2016 & $\begin{array}{l}\text { Außenminister Witold Waszczykowski trifft sich in Warschau mit seinem französischen Amtskollegen Jean- } \\
\text { Marc Ayrault. Bekräftigt wird der Wille zur Intensivierung der bilateralen Zusammenarbeit sowie der Zusam- } \\
\text { menarbeit im Rahmen des Weimarer Dreiecks (Polen, Frankreich, Deutschland). In Hinblick auf den von } \\
\text { Polen ausgerichteten NATO-Gipfel in Warschau im Juli unterstreichen die Minister die Notwendigkeit, dass } \\
\text { die NATO als solidarische Einheit auftritt. }\end{array}$ \\
\hline 14.06 .2016 & $\begin{array}{l}\text { Die Regierung beschließt die Anhebung des Mindestlohns von gegenwärtig } 1.850 \text { Zloty auf } 2.000 \text { Zloty } \\
\text { (ca. } 450 \text { Euro). }\end{array}$ \\
\hline 14.06 .2016 & $\begin{array}{l}\text { Aus Anlass der Feier des } 25 \text {-jährigen Bestehens des Deutsch-Polnischen Jugendwerks (DPJW) in Warschau for- } \\
\text { dert der Polen-Koordinator der Bundesregierung, Brandenburgs Ministerpräsident Dietmar Woidke, eine bes- } \\
\text { sere finanzielle Ausstattung der Institution, die seit Jahren unterfinanziert sei. }\end{array}$ \\
\hline 14.06 .2016 & $\begin{array}{l}\text { Die Verteidigungsminister der NATO-Staaten beschließen die Stationierung von jeweils einem Bataillon mit } \\
\text { etwa } 1.000 \text { Soldaten in Polen, Litauen, Lettland und Estland zur Stärkung der Ostflanke vor dem Hintergrund } \\
\text { des russisch-ukrainischen Konflikts. }\end{array}$ \\
\hline 15.06.2016 & $\begin{array}{l}\text { Der Senat berät über das Antiterrorgesetz, das der Sejm in der vergangenen Woche verabschiedet hat. Die Bür- } \\
\text { gerplattform (Platforma Obywatelska - PO) spricht sich für die Ablehnung des Gesetzes ohne Korrekturen aus. } \\
\text { Gravierende Mängel seien der zu umfangreiche Zugang der Agentur für Innere Sicherheit (Agencja Bezpieczeń- } \\
\text { stwa Wewnętrznego - ABW) zu den persönlichen Daten der Bürger in staatlichen Datenbanken und die Mög- } \\
\text { lichkeit, drei Monate lang ohne gerichtliche Anordnung operative Techniken gegenüber Ausländern einzuset- } \\
\text { zen. Recht und Gerechtigkeit (Prawo i Sprawiedliwość - PiS) vertritt den Standpunkt, dass eine Beschneidung } \\
\text { der Menschenrechte zugunsten der Sicherheit der Bürger vertretbar sei. }\end{array}$ \\
\hline 16.06 .2016 & $\begin{array}{l}\text { Im Rahmen der Feierlichkeiten zur Unterzeichnung des Vertrags über gute Nachbarschaft und freundschaftli- } \\
\text { che Zusammenarbeit zwischen Deutschland und Polen vor } 25 \text { Jahren empfängt Bundespräsident Joachim Gauck } \\
\text { in Berlin Präsident Andrzej Duda. Gauck weist darauf hin, dass in Westeuropa bei manchen die Erwartung } \\
\text { festzustellen sei, dass die Gesellschaften der ostmitteleuropäischen Länder dieselben kulturellen Standards und } \\
\text { Normen vertreten wie die westlichen, obgleich die Bedingungen, die die Gesellschaften geprägt haben, unter- } \\
\text { schiedlich gewesen seien. Auch im Rahmen der Europäischen Union bräuchten die Gesellschaften Freiräume, } \\
\text { um ihren eigenen Weg im zusammenwachsenden Europa zu finden. Duda sagt, er sei stolz, dass die polnische } \\
\text { und die deutsche Nation den Weg der Versöhnung gegangen seien. Der bilaterale Vertrag sei nicht nur eine poli- } \\
\text { tische Errungenschaft, sondern entspringe auch dem Wunsch beider Gesellschaften nach Annäherung. Die enge } \\
\text { Zusammenarbeit in gegenseitigem Respekt erlaube, eine starke Europäische Union aufzubauen. }\end{array}$ \\
\hline
\end{tabular}




\begin{tabular}{|c|c|}
\hline 16.06 .2016 & $\begin{array}{l}\text { Bei der Fußballeuropameisterschaft in Frankreich trennen sich die Nationalmannschaften Deutschlands und } \\
\text { Polens mit 0:0. }\end{array}$ \\
\hline 17.06.2016 & $\begin{array}{l}\text { Aus Anlass der Unterzeichnung des Vertrags über gute Nachbarschaft und freundschaftliche Zusammenar- } \\
\text { beit zwischen Deutschland und Polen vor } 25 \text { Jahren empfängt Bundeskanzlerin Angela Merkel in Berlin Prä- } \\
\text { sident Andrzej Duda. Merkel betont, dass die Erfolgsgeschichte der deutsch-polnischen Beziehungen keine } \\
\text { Selbstverständlichkeit sei und eine Verpflichtung für ihre gute Pflege bestünde. Die guten Beziehungen wür- } \\
\text { den die Grundlage schaffen, sich über Meinungsverschiedenheiten konstruktiv auszutauschen. Duda bezeich- } \\
\text { net die bilateralen Beziehungen als vorbildlich für die ganze Welt. Auch seien sie ein Fundament für die Euro- } \\
\text { päische Union, deren Krisen gemeinsam angegangen werden und für die Lösungen gefunden werden müssten, } \\
\text { die für alle akzeptabel seien. }\end{array}$ \\
\hline 17.06.2016 & $\begin{array}{l}\text { Präsident Andrzej Duda empfängt in Warschau Bundespräsident Joachim Gauck. Der Besuch gehört zu der } \\
\text { Reihe bilateraler Begegnungen anlässlich des } 25 \text {-jährigen Bestehens des deutsch-polnischen Vertrags über gute } \\
\text { Nachbarschaft und freundschaftliche Zusammenarbeit. Ein Themenschwerpunkt des Besuchs ist die Zukunft } \\
\text { der Europäischen Union. }\end{array}$ \\
\hline 18.06 .2016 & $\begin{array}{l}\text { Das Büro für Nationale Sicherheit (Biuro Bezpieczeństwa Narodowego - BBN) teilt mit, dass die Polnische } \\
\text { Armee zwei Kontingente zur Unterstützung des Bündnisses gegen den sogenannten Islamischen Staat entsen- } \\
\text { den wird. Die polnischen Soldaten werden nicht an Kampfeinsätzen teilnehmen. }\end{array}$ \\
\hline 19.06.2016 & $\begin{array}{l}\text { In Plock (Płock) nimmt Präsident Andrzej Duda an der Feier zum 40. Jahrestag der Arbeiterunruhen im Juni } \\
1976 \text { teil. Erhebungen fanden auch in Radom, Ursus und anderen Städten statt und führten schließlich zur } \\
\text { Gründung der oppositionellen Organisation Komitee zur Verteidigung der Arbeiter (Komitet Obrony Robot- } \\
\text { ników - KOR), das die Entstehung der Solidarność-Bewegung } 1980 \text { mit vorbereitete. }\end{array}$ \\
\hline 20.06 .2016 & $\begin{array}{l}\text { Der Staatspräsident der Volksrepublik China, Xi Jinping, beginnt seinen zweitägigen offiziellen Besuch in Polen. } \\
\text { Vorgesehen ist die Unterzeichnung verschiedener bilateraler Verträge über Zusammenarbeit in den Bereichen } \\
\text { Wirtschaft, Wissenschaft und Kultur. }\end{array}$ \\
\hline
\end{tabular}

Sie können die gesamte Chronik seit 2007 auch auf <http://www.laender-analysen.de/polen/> unter dem Link »Chronik«lesen. 


\section{ÜBER DIE POLEN-ANALYSEN}

Die Polen-Analysen erscheinen zweimal monatlich als E-Mail-Dienst. Sie werden gemeinsam vom Deutschen PolenInstitut Darmstadt, der Bremer Forschungsstelle Osteuropa und der Deutschen Gesellschaft für Osteuropakunde herausgegeben.

Ein Archiv der Polen-Analysen finden Sie im Internet unter <www.laender-analysen.de/polen>

Kostenloses Abonnement unter <http://www.deutsches-polen-institut.de/Newsletter/subscribe.php >

Diese Analysen finden Sie online als Lizenzausgabe auf

$<$ bpb.de>

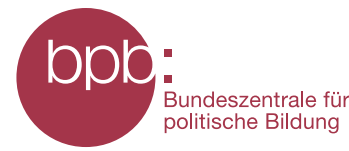

Deutsches Polen-Institut Darmstadt (<www.deutsches-polen-institut.de $>$ )

Das seit 1980 tätige Deutsche Polen-Institut Darmstadt (DPI) ist ein Forschungs-, Informations- und Veranstaltungszentrum für polnische Kultur, Geschichte, Politik, Gesellschaft und die deutsch-polnischen Beziehungen, die sich im Kontext der europäischen Integration entwickeln. Institutionelle Träger des DPI sind das Land Hessen, die Kultusminister der Länder, das Auswärtige Amt und die Wissenschaftsstadt Darmstadt. Einen wesentlichen Beitrag zur Verwirklichung der Institutsziele leisten private Stiftungen. Ziel der Vermittlertätigkeit des DPI ist es, »die zu interessieren, auf die es politisch, wirtschaftlich, gesellschaftlich und kulturell im deutsch-polnischen Verhältnis ankommt (Leitlinien 1997). Es geht um die Entscheider und Multiplikatoren in Politik, Kultur, Bildung, Verwaltung, Medien und Wirtschaft. Das DPI versteht sich in Kooperation mit den Orten wissenschaftlicher Polen-Kompetenz an deutschen Hochschulen und Forschungsinstituten als verbindendes und vernetzendes Zentrum. Mit der über 68.000 Bände zählenden multidisziplinären Fachbibliothek für Polen, die eine einzigartige Sammlung polnischer Belletristik in der Originalsprache und in deutscher Übersetzung umfasst, ist das DPI ein geschätzter Ort der Recherche und des wissenschaftlichen Arbeitens.

Forschungsstelle Osteuropa an der Universität Bremen (<www.forschungsstelle.uni-bremen.de>)

1982 gegründet, widmet sich die Forschungsstelle Osteuropa an der Universität Bremen der interdisziplinären Analyse der Länder Ost- und Ostmitteleuropas in Zeitgeschichte und Gegenwart. Der Forschungsschwerpunkt liegt dabei auf der Rolle von »Dissens und Konsens«, von Opposition und Zivilgesellschaft in ihrem historischen, politischen, gesellschaftlichen und kulturellen Kontext. Die Forschungsstelle besitzt in ihrem Archiv eine einzigartige Sammlung alternativer Kulturgüter und unabhängiger Texte aus den ehemaligen sozialistischen Ländern. Darunter befindet sich auch eine umfangreiche Sammlung des "Zweiten Umlaufs«, die das Schrifttum und Dokumente unabhängiger Initiativen und gesellschaftlicher Gruppen in Polen aus der Zeit von 1976 bis zum Umbruch umfasst. Hinzu kommt eine umfangreiche Bibliothek mit wissenschaftlicher Literatur. Mit Archiv, Bibliothek und zwei wissenschaftlichen Abteilungen ist die Forschungsstelle auch eine Anlaufstelle sowohl für Gastwissenschaftler als auch für die interessierte Öffentlichkeit.

Eine der Hauptaufgaben der Forschungsstelle ist die Information der interessierten Öffentlichkeit. Dazu gehören unter anderem regelmäßige E-Mail-Informationsdienste für Politik, Wirtschaft, Zivilgesellschaft und Medien.

Das Institut für Öffentliche Angelegenheiten (Instytut Spraw Publicznych - ISP) in Warschau ist einer der führenden Think Tanks in Polen und seit 1995 als unabhängiges Forschungszentrum zu grundlegenden Fragen des öffentlichen Lebens tätig. Das ISP kooperiert eng mit zahlreichen Experten und Forschern wissenschaftlicher Einrichtungen im In- und Ausland. <www.isp.org.pl>

Herausgegeben mit finanzieller Unterstützung der Stiftung für deutsch-polnische Zusammenarbeit
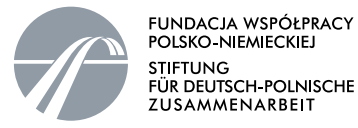

Die Meinungen, die in den Polen-Analysen geäußert werden, geben ausschließlich die Auffassung der Autoren wieder. Abdruck und sonstige publizistische Nutzung sind nach Rücksprache mit der Redaktion gestattet. Redaktion: Prof. Dr. Dieter Bingen (verantwortlich) (Darmstadt), Silke Plate M.A. (Bremen) Technische Gestaltung: Matthias Neumann

Polen-Analysen-Layout: Cengiz Kibaroglu, Matthias Neumann

Alle Ausgaben der Polen-Analysen sind mit Themen- und Autorenindex archiviert unter www.laender-analysen.de

Die Polen-Analysen werden im Rahmen der Datenbank World Affairs Online (WAO) ausgewertet und sind im Portal IREON www.ireon-portal.de recherchierbar.

ISSN 1863-9712 @ 2016 by Deutsches Polen-Institut Darmstadt und Forschungsstelle Osteuropa, Bremen

Kontakt: Dr. Andrzej Kaluza, Presse- und Öffentlichkeitsarbeit, Deutsches Polen-Institut, Residenzschloss, Marktplatz 15,

D-64283 Darmstadt, Tel.: +49/6151/4202-20, Fax: +49/6151/4202-10, E-Mail: polen-analysen@dpi-da.de, Internet: www.laender-analysen.de/polen 